\title{
Echoes from quantum confinement
}

The discovery of intrinsic quantum confinement effects in the form of oscillations in the optical absorption of formamidinium lead triiodide thin films is a vivid example of the surprising physical properties of these hybrid organic-inorganic materials.

\section{Alejandro R. Goñi}

Beyond their excellent photovoltaic performance, hybrid organic-inorganic metal halide perovskites are in the focus of intense fundamental research because of the unique physical phenomena and emerging functionalities prompted by the interplay between organic and inorganic components of these materials. ${ }^{1}$ The inorganic framework is composed of cornersharing metal halide octahedrons forming a labile and deformable structure, whereas the organic cations exhibit a rich rotational dynamics inside the cage voids. The size of the organic cations and their interaction with the inorganic framework determine the final crystal structure adopted by the hybrid perovskites at different temperatures. ${ }^{2}$ Moreover, pronounced lattice anharmonicity and strong electron-phonon coupling effects are observed which, for instance, lead to an extremely low thermal conductivity more familiar to amorphous polymers, ${ }^{3}$ and polaron-mediated electrical transport (polarons are charge carriers dressed with lattice vibrations), ${ }^{4}$ respectively. Writing in this issue of Nature Materials, ${ }^{5}$ Laura Herz and colleagues now uncovered another interesting feature of these materials - signatures of unintentional quantum confinement in a nominally bulk film - which highlights how the peculiar structural properties of hybrid lead halide perovskites at a microscopic level impact its macroscopic response in unprecedented ways.

Herz and collaborators observed the presence of well-defined, above-bandgap oscillations in the absorption spectra of formamidinium lead triiodide $\left(\mathrm{FAPbl}_{3}\right)$ thin films, as illustrated in Fig. 1. Interestingly, the oscillations were equally apparent for films of very different thicknesses ranging from 35 to $850 \mathrm{~nm}$, thus ruling out spurious effects such as optical interferences. In contrast, only monotonous absorption profiles were recorded either for thinner films $(10 \mathrm{~nm}$ thick) or for other hybrid perovskites like the archetypal methylammonium lead triiodide. The relevance of this work resides in that the discrete features can be interpreted as manifestation of intrinsic quantum confinement effects, unintentionally occurring in the $\mathrm{FAPbl}_{3}$ thin films. The researchers arrived at this conclusion after a careful and in-depth analysis of the temperature and peak-index dependence of the feature energies, among other experiments, supported by ab-initio simulations of the absorption profiles. As illustrated by the sketches in the inset to Fig. 1, in a broad sense, quantum confinement acts on the electronic band structure either by discretization of the energy spectrum due to full restriction of the movement of a particle confined in deep wells (infinite potential barrier model) or by leading to mini-bands formation, in case the (finite) confining potential exhibits periodicity. These changes in the electronic landscape lead to peaks in the joint density of states, as probed in absorption. Remarkably, the confining potential does not seem to impair the electrical transport in the thin films. 
Like with other semiconductors, it is possible to physically confine metal halide perovskites in domains comparable with the material's exciton Bohr radius, either by post-deposition nanostructuration or by synthesizing colloidal nanocrystals of nanometric size. This strategy is used to tailor the physical properties of perovskites and their performance in devices. ${ }^{6,7}$ However, the films deposited by this research team are not intentionally nanostructured, yet they show quantum confinement effects. Hence, a challenge of this work is to pinpoint the precise origin of the confining potential. One possibility offered is the spontaneous formation of ferroelastic or polar ferroelectric domains. However, ferroelectricity is currently an intensely controversial topic for $\mathrm{MAPbl}_{3}$ and the existence of ferroelectric as well as ferroelastic domains in the cubic room-temperature phase of $\mathrm{FAPbl}_{3}$ is more than arguable. A second mechanism suggested by the researchers is the formation of nanoscale inclusions of the so-called $\delta$-phase of $\mathrm{FAPbl}_{3}$, a semiconducting hexagonal phase with a much larger gap and slightly more stable at room temperature than the perovskitic $\alpha$-phase. The coexistence of $\alpha$-phase domains (as quantum wells) surrounded by $\delta$-phase inclusions (as barriers) appears as a plausible mechanism to produce the necessary quantum confinement effects, ultimately leading to the oscillatory features uncovered here in the absorption spectra of $\mathrm{FAPbl}_{3}$ thin films. The polymorphism of $\mathrm{FAPbl}_{3}$ results from the somewhat large size of the FA cation, yet it is also affected by external factors. For instance, it has been shown that the use of external additives like $\mathrm{PbS}$ colloidal quantum dots can alter the structural stability of $\mathrm{FAPbl}_{3}$, varying the formation energy of the different phases as a consequence of several effects taking place at the perovskite/additive interface: strain build-up, changes in the surface energy and chemical bonding between both materials. ${ }^{8}$ These competing mechanisms might be also at work at the interface between the substrate and the $\mathrm{FAPbl}_{3}$ film, leading to quantum confinement by unintentional formation of $\alpha$-phase inclusions surrounded by thin $\delta$-phase layers. This could also suggest a strategy to control the confinement potential in $\mathrm{FAPbl}_{3}$ by proper pre-patterning of the substrate. It remains to be seen whether further investigations will confirm this interpretation, and whether the concept can be extended to other members of the hybrid perovskite family.

The discovery of the phenomenon of absorption oscillations related to intrinsic quantumconfinement effects by Herz and coworkers highlights once more the rich physics inherent to hybrid perovskites. Understanding the mechanisms leading to such phenomenon may suggest new routes for manipulating their electronic band structure at the nanoscale, in order to enhance optoelectronic performance by exploiting the confinement-induced discretization of the energy spectrum. Strikingly, material nanostructuration is achieved in a natural, less cumbersome manner as compared with conventional top-down techniques, paving the way to a cost-effective technology based on hybrid perovskites.

\author{
Alejandro R. Goñi ${ }^{1,2}$ \\ ${ }^{1}$ ICREA, Barcelona, Spain. \\ ${ }^{2}$ Institute of Materials Science of Barcelona (ICMAB-CSIC), Bellaterra, Spain. \\ e-mail: goni@icmab.es
}






Figure 1| Signatures of intrinsic quantum confinement in $\mathrm{FAPbl}_{3}$. Above-gap oscillations observed in the absorption spectra of $\mathrm{FAPbl}_{3}$ thin films at different temperatures. The inset shows two concurrent mechanisms that may lead to the oscillations: strict quantum confinement in deep wells and the periodicity of a superlattice confining potential. Black lines represent the spatial distribution of the energy potential; red lines represent the discrete energy levels or minibands formed in the electronic band structure of the material. Figure adapted from ref. 5.

\section{References}

1. Whalley, L.D., Frost, J.M., Jung, Y.-K. \& Walsh, A. J. Chem. Phys. 146, 220901 (2017).

2. Ghosh, D., Atkins, P.W., Islam, M.S., Walker, A.B. \& Eames, C. ACS Energy Lett. 2, 2424-2429 (2017).

3. Pisoni, A., Jaćimović, J., Barišić, O.S., Spina, M., Gaál, R., Forró, L. \& Horváth, E. J. Phys. Chem. Lett. 5, 2488-2492 (2014).

4. Ghosh, D., Welch, E., Neukirch, A.J., Zakhidov, A. \& Tretiak, S. J. Phys. Chem. Lett. 11, 3271-3286 (2020).

5. Wright, A.D., Volonakis, G., Borchert, J., Davies, C.L., Giustino, F., Johnston, M.B. \& Herz, L.M. Nat. Mater. https://doi.org/10.1038/s41563-020-0774-9 (2020).

6. Protesescu, L., Yakunin, S., Bodnarchuk, M.I., Krieg, F., Caputo, R., Hendon, C.H., Yang, R.X., Walsh, A. \& Kovalenko, M.V. Nano Lett. 15, 3692-3696 (2015).

7. Fang, H.-H., Protesescu, L., Balasz, D.M., Adjokatse, S., Kovalenko, M.V. \& Loi, M.A. Small 13, 1700673 (2017).

8. Masi, S., Echeverría-Arrondo, C., Salim, K.M.M., Ngo, T.T., Mendez, P.F., López-Fraguas, E., MaciasPinilla, D.F., Planelles, J., Climente, J.I. \& and Mora-Seró, I. ACS Energy Lett. 5, 418-427 (2020). 